International Electronic Journal of Geometry

Volume 8 No. 1 PP. 116-127 (2015) (CIEJG

\title{
TIMELIKE SURFACES OF REVOLUTION WITH CONSTANT MEAN CURVATURE IN DE SITTER 3-SPACE
}

\author{
SUNGWOOK LEE AND JACOB MARTIN \\ (Communicated by Cihan ÖZGÜR)
}

\begin{abstract}
In this paper, we construct timelike surfaces of revolution with constant mean curvature $H=c$ and minimal timelike surfaces of revolution in de Sitter 3-space $\mathbb{S}_{1}^{3}\left(c^{2}\right)$ of constant sectional curvature $c^{2}$. It is shown that timelike surfaces of revolution with constant mean curvature $H=c$ in $\mathbb{S}_{1}^{3}\left(c^{2}\right)$ tend toward a timelike catenoid, the minimal timelike surface of revolution in Minkowski 3-space $\mathbb{R}^{2+1}$ as $c \rightarrow 0$. Minimal timelike surfaces of revolution in $\mathbb{S}_{1}^{3}\left(c^{2}\right)$ also tend toward the timelike catenoid in $\mathbb{R}^{2+1}$ as $c \rightarrow 0$.
\end{abstract}

\section{INTRODUCTION}

The symmetry group of the Minkowski 3 -space $\mathbb{R}^{2+1}$ is the Lorentz group $\mathrm{O}(2,1)$, i.e. the set of all Lorentz isometries of $\mathbb{R}^{2+1}$. In particular, the parity preserving Lorentz isometries form a subgroup $\mathrm{SO}(2,1)$ of $\mathrm{O}(2,1)$ which is called the special Lorentz group. $\mathrm{SO}(2,1)$ contains rotations about the time axis and Lorentz boosts which may be considered as rotations about spacial axis in $\mathbb{R}^{2+1}$. In physics, the trajectory of a massive particle is spacetime is called a worldline. In geometry, a worldline $x(\lambda)=\left(x^{\mu}(\lambda)\right)$ is called a timelike curve. The velocity vector $\frac{d x^{\mu}}{d \lambda}$ satisfies $\langle x(\lambda), x(\lambda)\rangle=\eta_{\mu \nu} \frac{d x^{\mu}}{d \lambda} \frac{d x^{\nu}}{d \lambda}<0$, where $\langle$,$\rangle is the inner product induced by the flat$ Lorentzian metric of $\mathbb{R}^{2+1}$ and $\eta_{\mu \nu}$ is the metric tensor of signature $(-++)$. The arc-length of $x(\lambda)$ is defined to be

$$
\triangle x:=\int \sqrt{-\eta_{\mu \nu} \frac{d x^{\mu}}{d \lambda} \frac{d x^{\nu}}{d \lambda}} d \lambda .
$$

In physics, $x(\lambda)$ is called proper time and is interpreted as the actual time elapsed on a physical clock carried along the curve. A surface that is obtained by rotating a timelike curve about an axis in $\mathbb{R}^{2+1}$ is a timelike surface of revolution. In [5], the authors studied on how to construct a timelike surface of revolution with constant mean curvature in $\mathbb{R}^{2+1}$ by solving the differential equation of its profile curve. Other than flat Minkowski 3-space, there is another interesting Lorentzian

Date: Received: June 11, 2014 and Accepted: March 06, 2015

2000 Mathematics Subject Classification. 53A10, 53C42, 53C50.

Key words and phrases. constant mean curvature, de Sitter 3-space, flat chart model, Lorentz surface, minimal surface, rotational surface, surface of revolution, timelike surface. 
3-manifold called de Sitter 3 -space $\mathbb{S}_{1}^{3}\left(c^{2}\right)$. de Sitter 3 -space is a timelike manifold of constant sectional curvature $c^{2}$. de Sitter 3 -space has $\mathrm{SO}(2)$ symmetry, so we may still consider rotations there. In this paper, the authors consider the flat chart model of $\mathbb{S}_{1}^{3}\left(c^{2}\right)$ which is $\mathbb{R}^{3}(t, x, y)$ with the metric $g_{c}=-(d t)^{2}+e^{2 c t}\left\{(d x)^{2}+(d y)^{2}\right\}$. From the metric we clearly see that rotations in the $x y$-plane are the only kind of rotations that may be considered in $\mathbb{S}_{1}^{3}\left(c^{2}\right)$. The authors study how to construct timelike surfaces of constant mean curvature in $\mathbb{S}_{1}^{3}\left(c^{2}\right)$. From a string theory point of view, timelike surfaces of revolution with constant mean curvature in $\mathbb{S}_{1}^{3}\left(c^{2}\right)$ may be regarded as string worldsheets that are swept by closed strings in $\mathbb{S}_{1}^{3}\left(c^{2}\right)$.

Due to an analogue of Lawson correspondence ([3]) discussed in [4], there is a one-to-one correspondence between timelike surfaces of constant mean curvature $H_{s}$ in $\mathbb{S}_{1}^{3}\left(c^{2}\right)$ and timelike surfaces of constant mean curvature $H_{m}= \pm \sqrt{H_{s}^{2}+c^{2}}$ in $\mathbb{R}^{2+1}$. So, under the Lawson type correspondence, there are no timelike surfaces of constant mean curvature in $\mathbb{S}_{1}^{3}\left(c^{2}\right)$ that are corresponded to minimal timelike surfaces in $\mathbb{R}^{2+1}$. Interestingly, however, we show in this paper that the timelike catenoid, the minimal timelike surface of revolution in $\mathbb{R}^{2+1}$ is the limit of timelike surfaces of revolution with constant mean curvature $H=c$ in $\mathbb{S}_{1}^{3}\left(c^{2}\right)$ as $c \rightarrow 0$.

The authors also study how to construct minimal timelike surfaces of revolution in $\mathbb{S}_{1}^{3}\left(c^{2}\right)$ using calculus of variations. Note that here the harmonic map equation is no longer the wave equation, so minimal timelike surfaces in $\mathbb{S}_{1}^{3}\left(c^{2}\right)$ is not characterized by their mean curvature. We construct minimal timelike surfaces of revolution in $\mathbb{S}_{1}^{3}\left(c^{2}\right)$ using the calculus of variations. The minimal timelike surfaces of revolution in $\mathbb{S}_{1}^{3}\left(c^{2}\right)$ also tend toward the spacelike catenoid in $\mathbb{R}^{2+1}$ as $c \rightarrow 0$.

The second named author Jacob was a promising high school (Oak Grove High School) senior when he joined the first named author for the research project reported in this paper. The research has been done during Summer 2013-Fall 2013. Jacob is currently an undergraduate student majoring mathematics at Massachusetts Institute of Technology. The Department of Mathematics at the University of Southern Mississippi is committed to support nearby high schools by providing talented high school students opportunities for a research experience in mathematical sciences. This research was done as part of such an outreach effort.

\section{The Flat Chart Model of De Sitter 3-Space $\mathbb{S}_{1}^{3}\left(c^{2}\right)$}

Let $\mathbb{R}^{3+1}$ denote Minkowski spacetime with rectangular coordinates $x^{0}, x^{1}, x^{2}$, $x^{3}$ and the Lorentzian metric

$$
d s^{2}=-\left(d x^{0}\right)^{2}+\left(d x^{1}\right)^{2}+\left(d x^{2}\right)^{2}+\left(d x^{3}\right)^{2} .
$$

De Sitter 3-space is the hyperquadric

$$
\mathbb{S}^{3}\left(c^{2}\right):=\left\{\left(x^{0}, x^{1}, x^{2}, x^{3}\right) \in \mathbb{R}^{3+1}:-\left(x^{0}\right)^{2}+\left(x^{1}\right)^{2}+\left(x^{2}\right)^{2}+\left(x^{3}\right)^{2}=\frac{1}{c^{2}}\right\}
$$

which is a 3-dimensional hyperboloid of one sheet in spacetime. De Sitter 3-space is a timelike 3-manifold of constant sectional curvature $c^{2}$. Consider the open chart

$$
U=\left\{\left(x^{0}, x^{1}, x^{2}, x^{3}\right) \in \mathbb{S}^{3}\left(c^{2}\right): x^{0}+x^{1}>0\right\}
$$


and define (see [2])

$$
\begin{aligned}
t & =\frac{1}{c} \log c\left(x^{0}+x^{1}\right), \\
x & =\frac{x^{2}}{c\left(x^{0}+x^{1}\right)}, \\
y & =\frac{x^{3}}{c\left(x^{0}+x^{1}\right)} .
\end{aligned}
$$

Then

$$
d s^{2}=-(d t)^{2}+e^{2 c t}\left\{(d x)^{2}+(d y)^{2}\right\} .
$$

$\mathbb{R}^{3}$ with coordinates $t, x, y$ and the metric

$$
g_{c}:=-(d t)^{2}+e^{2 c t}\left\{(d x)^{2}+(d y)^{2}\right\}
$$

is called the flat chart model of de Sitter 3 -space. We will still denote it by $\mathbb{S}^{3}\left(c^{2}\right)$. As $c \rightarrow 0, \mathbb{S}^{3}\left(c^{2}\right)$ flattens out to Minkowski 3-space $\mathbb{R}^{2+1}$.

\section{Parametric Timelike Surfaces in $\mathbb{S}^{3}\left(c^{2}\right)$}

Let $M$ be a domain ${ }^{1}$ and $\varphi: M \longrightarrow \mathbb{S}^{3}\left(c^{2}\right)$ an immersion. The metric (1.4) induces an inner product $\langle$,$\rangle on each tangent space T_{p} \mathbb{S}^{3}\left(c^{2}\right)$.

Definition 2.1. An immersion $\varphi: M(u, v) \longrightarrow \mathbb{S}^{3}\left(c^{2}\right)$ is said to be timelike if $\frac{\partial \varphi}{\partial u}$ is timelike and $\frac{\partial \varphi}{\partial v}$ is spacelike, i.e.

$$
\left\langle\frac{\partial \varphi}{\partial u}, \frac{\partial \varphi}{\partial u}\right\rangle<0,\left\langle\frac{\partial \varphi}{\partial v}, \frac{\partial \varphi}{\partial v}\right\rangle>0
$$

Using the induced inner product on each $T_{p} \mathbb{S}^{3}\left(c^{2}\right)$, we can speak of conformal surfaces in $\mathbb{S}^{3}\left(c^{2}\right)$.

Definition 2.2. $\varphi: M \longrightarrow \mathbb{S}^{3}\left(c^{2}\right)$ is said to be Lorentz conformal if

$$
\begin{array}{r}
\left\langle\varphi_{u}, \varphi_{v}\right\rangle=0, \\
-\left|\varphi_{u}\right|^{2}=\left|\varphi_{v}\right|^{2}=e^{\omega},
\end{array}
$$

where $(u, v)$ is a local coordinate system in $M$ and $\omega: M \longrightarrow \mathbb{R}$ is a real-valued function in $M$. Lorentz conformal timelike surfaces are called Lorentz surfaces.

The induced metric on the Lorentz surface is given by

$$
d s_{\varphi}^{2}=\langle d \varphi, d \varphi\rangle=e^{\omega}\left\{-(d u)^{2}+(d v)^{2}\right\} .
$$

If $N$ is a unit normal vector field of a timelike immersion $\varphi: M \longrightarrow \mathbb{S}_{1}^{3}\left(c^{2}\right)$, then

$$
\langle N, N\rangle=1,\left\langle N, \varphi_{u}\right\rangle=\left\langle N, \varphi_{v}\right\rangle=0 .
$$

In order to calculate a unit normal vector field, we need an analogue of the cross product. We will still call that analogue the cross product. Although $\mathbb{S}_{1}^{3}\left(c^{2}\right)$ is not a vector space, the cross product on it can be defined locally on each tangent space $T_{p} \mathbb{S}_{1}^{3}\left(c^{2}\right)$, which is a vector space. Let $\mathbf{v}=v_{1}\left(\frac{\partial}{\partial t}\right)_{p}+v_{2}\left(\frac{\partial}{\partial x}\right)_{p}+v_{3}\left(\frac{\partial}{\partial y}\right)_{p}$, $\mathbf{w}=w_{1}\left(\frac{\partial}{\partial t}\right)_{p}+w_{2}\left(\frac{\partial}{\partial x}\right)_{p}+w_{3}\left(\frac{\partial}{\partial y}\right)_{p} \in T_{p} \mathbb{S}^{3}\left(c^{2}\right)$, where $\left\{\left(\frac{\partial}{\partial t}\right)_{p},\left(\frac{\partial}{\partial x}\right)_{p},\left(\frac{\partial}{\partial y}\right)_{p}\right\}$

\footnotetext{
${ }^{1}$ A 2-dimensional connected open set.
} 
denote the canonical basis for $T_{p} \mathbb{S}^{3}\left(c^{2}\right)$. Then the cross product $\mathbf{v} \times \mathbf{w}$ is defined to be

$$
\begin{aligned}
\mathbf{v} \times \mathbf{w}=\left(-v_{2} w_{3}+v_{3} w_{2}\right)\left(\frac{\partial}{\partial t}\right)_{p} & +e^{-2 c t}\left(v_{3} w_{1}-v_{1} w_{3}\right)\left(\frac{\partial}{\partial x}\right)_{p} \\
& +e^{-2 c t}\left(v_{1} w_{2}-v_{2} w_{1}\right)\left(\frac{\partial}{\partial y}\right)_{p}
\end{aligned}
$$

where $p=(t, x, y) \in \mathbb{S}^{3}\left(c^{2}\right)$.

We can also write (2.3) simply as a determinant

$$
\mathbf{v} \times \mathbf{w}=\left|\begin{array}{ccc}
-\frac{\partial}{\partial t} & e^{-2 c t} \frac{\partial}{\partial x} & e^{-2 c t} \frac{\partial}{\partial y} \\
v_{1} & v_{2} & v_{3} \\
w_{1} & w_{2} & w_{3}
\end{array}\right| .
$$

One may also define a triple scalar product $\langle\mathbf{u}, \mathbf{v} \times \mathbf{w}\rangle$ as a determinant

$$
\langle\mathbf{u}, \mathbf{v} \times \mathbf{w}\rangle=\left|\begin{array}{ccc}
-u_{1} & e^{-2 c t} u_{2} & e^{-2 c t} u_{3} \\
v_{1} & v_{2} & v_{3} \\
w_{1} & w_{2} & w_{3}
\end{array}\right| .
$$

However, the cross product and the inner product are not interchangeable i.e.

$$
\langle\mathbf{u}, \mathbf{v} \times \mathbf{w}\rangle \neq\langle\mathbf{u} \times \mathbf{v}, \mathbf{w}\rangle
$$

unlike the Euclidean case.

Let

$$
E:=\left\langle\varphi_{u}, \varphi_{u}\right\rangle, F:=\left\langle\varphi_{u}, \varphi_{v}\right\rangle, G:=\left\langle\varphi_{v}, \varphi_{v}\right\rangle .
$$

Proposition 2.1. Let $\varphi: M \longrightarrow \mathbb{S}^{3}\left(c^{2}\right)$ be an immersion. Then on each tangent plane $T_{p} \varphi(M)$,

$$
\left\|\varphi_{u} \times \varphi_{v}\right\|^{2}=e^{-4 c t(u, v)}\left(F^{2}-E G\right)
$$

where $p=(t(u, v), x(u, v), y(u, v)) \in \mathbb{S}^{3}\left(c^{2}\right)$.

Proof. Straightforward by a direct calculation.

Remark 2.1. If $c \rightarrow 0,(2.7)$ becomes the familiar formula in Lorentzian case [5]

$$
\left\|\varphi_{u} \times \varphi_{v}\right\|^{2}=F^{2}-E G .
$$

\section{The Mean curvature of a Parametric Surface in $\mathbb{S}^{3}\left(c^{2}\right)$}

In the well-known Euclidean case, the mean curvature of a parametric surface $\varphi(u, v)$ may be calculated by Gauss's beautiful formula [7]

$$
H=\frac{G \ell+E \mathfrak{n}-2 F \mathfrak{m}}{2\left(E G-F^{2}\right)}
$$

where

$$
\ell=\left\langle\varphi_{u u}, N\right\rangle, \mathfrak{m}=\left\langle\varphi_{u v}, N\right\rangle, \mathfrak{n}=\left\langle\varphi_{v v}, N\right\rangle
$$

and $N$ is the unit normal vector field of $\varphi(u, v)$. The formula (3.1) is still valid for parametric surfaces in any 3 -dimensional space-form including timelike surfaces in $\mathbb{S}_{1}^{3}\left(c^{2}\right)$. The derivation of (3.1) in [7] requires a use of Lagrange's identity which is no longer valid in tangent spaces of $\mathbb{S}^{3}\left(c^{2}\right)$. However, the formula (3.1) may also be proved using the shape operator of a parametric surface in a 3-dimensional space-form obtained from its unit normal vector field. The proof is elementary and 
may be found in [6] for instance. In particular, we obtain the following proposition from (3.1).

Proposition 3.1. Let $\varphi: M \longrightarrow \mathbb{S}^{3}\left(c^{2}\right)$ be a conformal surface satisfying (2.1). Then the mean curvature $H$ of $\varphi$ is computed to be

$$
H=\frac{1}{2} e^{-\omega}\langle\square \varphi, N\rangle,
$$

where $\square=-\frac{\partial^{2}}{\partial u^{2}}+\frac{\partial^{2}}{\partial v^{2}}$ is d'Alembert's operator.

\section{Lorentz Surfaces of Revolution with Constant Mean Curvature IN $\mathbb{S}^{3}\left(c^{2}\right)$}

There is an interesting one-to-one correspondence, the Lawson-Guichard correspondence, between constant mean curvature surfaces in different semi-Riemannian space forms [4]. The correspondence is more than just a bijection. Corresponding constant mean curvature surfaces satisfy the same Gauss-Codazzi equations, so they share many geometric properties in common, even though they live in different spaces. For this reason they are often called cousins. In particular, there is a one-to-one correspondence between timelike surfaces of constant mean curvature $H$ in $\mathbb{S}^{3}\left(c^{2}\right)$ and timelike surfaces of constant mean curvature ${ }^{2}$

$$
H_{m}= \pm \sqrt{H^{2}+c^{2}}
$$

in Minkowski 3-space $\mathbb{R}^{2+1}$. As seen clearly in (4.1), there are no timelike surfaces of constant mean curvature in $\mathbb{S}_{1}^{3}\left(c^{2}\right)$ that correspond to minimal ${ }^{3}$ timelike surfaces in $\mathbb{R}^{2+1}$. Physically, minimal timelike surfaces in $\mathbb{R}^{2+1}$ are bosonic string worldsheets. While there are no cousins in $\mathbb{S}_{1}^{3}\left(c^{2}\right)$ of minimal timelike surfaces in $\mathbb{R}^{2+1}$, the limit of timelike surfaces of revolution with constant mean curvature $H=c$ in $\mathbb{S}_{1}^{3}\left(c^{2}\right)$ as $c \rightarrow 0$ is the timelike catenoid ${ }^{4}$, the minimal timelike surface of revolution in $\mathbb{R}^{2+1}$.

In this section, we are interested in constructing a Lorentz surface of revolution with constant mean curvature $H=c$ in $\mathbb{S}^{3}\left(c^{2}\right)$ which corresponds to a Lorentz surface of constant mean curvature $\pm \sqrt{2} c$ in $\mathbb{R}^{2+1}$ via the Lawson-Guichard correspondence.

From the metric (1.4), one can see that $\mathbb{S}_{1}^{3}\left(c^{2}\right)$ has $\mathrm{SO}(2)$ symmetry, i.e. $\mathrm{SO}(2)$ is a subgroup of the isometry group of $\mathbb{S}_{1}^{3}\left(c^{2}\right)$, and it is the maximally rotational symmetry. More specifically, the rotations about the $t$-axis (i.e. rotations on the $x y$-plane) are the only type of Euclidean rotations that can be considered in $\mathbb{S}_{1}^{3}\left(c^{2}\right)$.

Consider a profile curve $\alpha(u)=(g(u), h(u), 0)$ in the $t x$-plane. Denote by $\varphi(u, v)$ the rotation of $\alpha(u)$ about $t$-axis through an angle $v$. Then

$$
\varphi(u, v)=(g(u), h(u) \cos v, h(u) \sin v) .
$$

If $\dot{g}(u)=\frac{d g(u)}{d u}$ is never $0,(4.2)$ has a parametrization of the form

$$
\varphi(w, v)=(w, f(w) \cos v, f(w) \sin v) .
$$

\footnotetext{
${ }^{2}$ The choice of \pm signs depends on the orientation of the surface.

${ }^{3}$ Area minimizing surfaces or equivalently conformal surfaces with zero mean curvature.

${ }^{4}$ Timelike catenoid is physically worldsheet of a closed string in $\mathbb{R}^{2+1}$.
} 
Thus, without loss of generality we may assume that $g(u)=u$ in (4.2). The quantities $E, F, G$ are calculated to be

$$
\begin{aligned}
& E=e^{2 c u}\left\{-e^{-2 c u}+\dot{h}(u)^{2}\right\}, \\
& F=0, \\
& G=e^{2 c u} h(u)^{2} .
\end{aligned}
$$

If we require $\varphi(u, v)$ to be conformal, then

$$
e^{-2 c u}-\dot{h}(u)^{2}=h(u)^{2} .
$$

The quantities $\ell, \mathfrak{m}, \mathfrak{n}$ are calculated to be

$$
\begin{aligned}
& \ell=-\frac{\ddot{h}(u) h(u)}{\sqrt{h(u)^{2}\left(e^{-2 c u}-\dot{h}(u)^{2}\right)}}, \\
& \mathfrak{m}=0, \\
& \mathfrak{n}=\frac{h(u)^{2}}{\sqrt{h(u)^{2}\left(e^{-2 c u}-\dot{h}(u)^{2}\right)}} .
\end{aligned}
$$

So the mean curvature $H$ is calculated by

$$
\begin{aligned}
H & =\frac{G \ell+E \mathfrak{n}-2 F \mathfrak{m}}{2\left(E G-F^{2}\right)} \\
& =\frac{1}{2} \frac{-h(u) \ddot{h}(u)-e^{-2 c u}+\dot{h}(u)^{2}}{e^{2 c u}\left(-e^{-2 c u}+\dot{h}(u)^{2}\right) \sqrt{h(u)^{2}\left(e^{-2 c u}-\dot{h}(u)^{2}\right)}} .
\end{aligned}
$$

With the conformality condition (4.3), $H$ becomes

$$
H=\frac{\ddot{h}(u)+h(u)}{2 e^{2 c u} h(u)^{3}} .
$$

Differentiating (4.3) with respect to $u$, we obtain

$$
\dot{h}(u)(\ddot{h}(u)+h(u))=c e^{2 c u} .
$$

It follows from (4.4) and (4.5) that if $H=0$ then $c=0$ and hence we have:

Proposition 4.1. There are no Lorentz surfaces of revolution with $H=0$ in $\mathbb{S}_{1}^{3}\left(c^{2}\right)$.

Remark 4.1. Although $\mathbb{S}_{1}^{3}\left(c^{2}\right)$ does not admit Lorentz surfaces of revolution with $H=0$, it does not mean that there are no timelike surfaces with $H=0$ in $\mathbb{S}_{1}^{3}\left(c^{2}\right)$. For instance, the timelike plane $(u, v, 0)$ in $\mathbb{S}_{1}^{3}\left(c^{2}\right)$ has $H=0$. The timelike plane is not conformal in $\mathbb{S}_{1}^{3}\left(c^{2}\right)$.

Remark 4.2. The timelike catenoid $\varphi(u, v)=(u, \cos u \cos v, \cos u \sin v)$ is a minimal Lorentz surface in $\mathbb{R}^{2+1}$ (see [5]). One may consider the timelike catenoid in $\mathbb{S}_{1}^{3}\left(c^{2}\right)$ but then it is not conformal since $E=-1+e^{2 c u} \sin ^{2} u, F=0$, and $G=e^{2 c u} \cos ^{2} u$. Its mean curvature is neither 0 nor constant. It is given by

$$
H=-\frac{1}{2} \frac{-1+e^{2 c u}}{|\cos u|\left(1-e^{2 c u} \sin ^{2} u\right)^{\frac{3}{2}}} .
$$

$\varphi(u, v)$ satisfies the equation $-\varphi_{u u}+\varphi_{v v}=0$. Note that this does not lead to $H=0$ since $\varphi(u, v)$ is not conformal. 
Let $H=c$. Then (4.4) can be written as

$$
\ddot{h}(u)+h(u)-2 c e^{2 c u} h(u)^{3}=0 .
$$

Hence, constructing a surface of revolution with $H=c$ comes down to solving the second order nonlinear differential equation (4.6). Unfortunately, we cannot solve (4.6) analytically, so we solve it numerically with the aid of MAPLE. (See Appendix $\mathrm{B}$ of [6] for details of the computational procedure.) In the next section, we show the graphics of the Lorentz surface of revolution with constant mean curvature $H=c$ in $\mathbb{S}_{1}^{3}\left(c^{2}\right)$ that we obtained using the numerical solution of the differential equation (4.6). The conformality condition (4.3) can be used to determine initial conditions. For all the numerical solutions of (4.6) in this paper, we used the same initial conditions $h(0)=0$ and $\dot{h}(0)=\sqrt{1-h(0)^{2}}=1$.

If $c \rightarrow 0$, then (4.6) becomes

$$
\ddot{h}(u)+h(u)=0
$$

which is an equation of underdamped simple harmonic oscillator. The equation (4.7) has the general solution

$$
h(u)=c_{1} \cos u+c_{2} \sin u .
$$

This $h(u)$ gives rise to a minimal timelike surface of revolution in $\mathbb{R}^{2+1}$ which is called a timelike catenoid. For $c_{1}=1, c_{2}=0, \varphi(u, v)$ is given by

$$
\varphi(u, v)=(u, \cos u \cos v, \cos u \sin v) .
$$

This is a minimal surface of revolution in $\mathbb{R}^{2+1}$ which is called a timelike catenoid. Figure 1 shows a timelike catenoid with $h(0)=0$ and $\dot{h}(0)=1$.

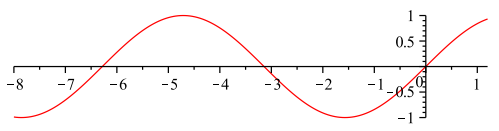

(a)

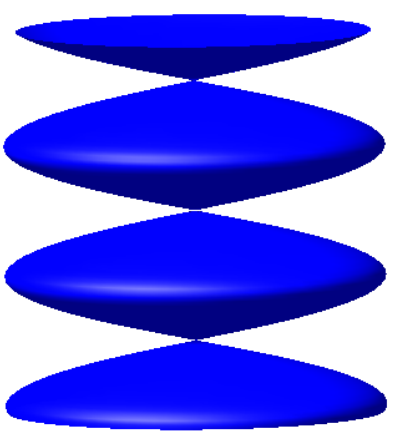

(b)

Figure 1. (a) Profile Curve $h(u),-8 \leq u \leq 1.2$, (b) Timelike Catenoid in $\mathbb{R}^{2+1}$

\section{The Illustration of the Limit of Lorentz Surfaces of Revolution WITH $H=c$ IN $\mathbb{S}_{1}^{3}\left(c^{2}\right)$ AS $c \rightarrow 0$}

In section 4 , it is shown that the limit of Lorentz surfaces of revolution with constant mean curvature $H=c$ in $\mathbb{S}_{1}^{3}\left(c^{2}\right)$ is the timelike catenoid, the minimal timelike surface of revolution in $\mathbb{R}^{2+1}$. In this section, such limiting behavior of 
Lorentz surfaces of revolution with $H=c$ in $\mathbb{S}_{1}^{3}\left(c^{2}\right)$ is illustrated with graphics in Figure $2(H=1)$, Figure $3\left(H=\frac{1}{2}\right)$, Figure $4\left(H=\frac{1}{4}\right)$, Figure $5\left(H=\frac{1}{8}\right)$, Figure $6\left(H=\frac{1}{64}\right)$, and Figure $7\left(H=\frac{1}{256}\right)$. These still images clearly show the limiting behavior of Lorentz surfaces of revolution with CMC $H=c$ in $\mathbb{S}_{1}^{3}\left(c^{2}\right)$ as $c \rightarrow 0$. The authors have made an animation of the limiting behavior available at http://www.math.usm.edu/lee/tldscmcanim.gif. The graphic of each profile curve $h(u)$ (in blue) is drawn with the graphic of the profile curve of timelike catenoid in $\mathbb{R}^{2+1}$ (in red) for visual comparison. The graphic of each Lorentz surface of revolution (in blue) is also drawn with the graphic of timelike catenoid in $\mathbb{R}^{2+1}$ (in transparent greyscale) for visual comparison.

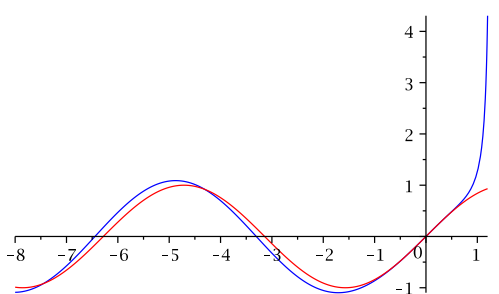

(a)

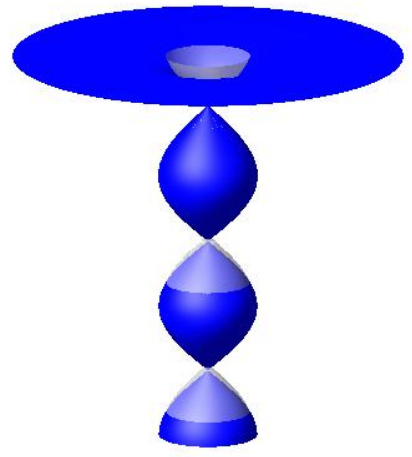

(b)

Figure 2. CMC $H=1$ : (a) Profile Curve $h(u),-8 \leq u \leq 1.2$, (b) Lorentz Surface of Revolution in $\mathbb{S}_{1}^{3}(1)$

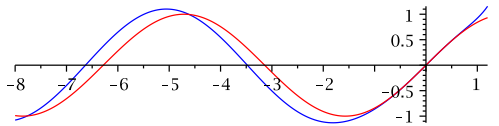

(a)

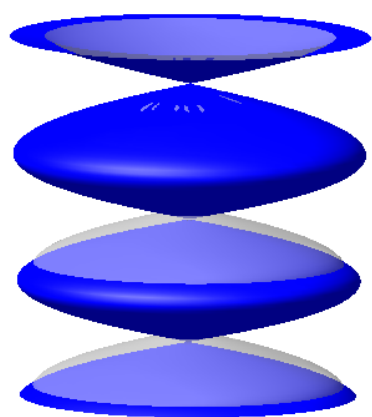

(b)

Figure 3. CMC $H=\frac{1}{2}$ : (a) Profile Curve $h(u),-8 \leq u \leq 1.2$,

(b) Lorentz Surface of Revolution in $\mathbb{S}_{1}^{3}\left(\frac{1}{4}\right)$ 


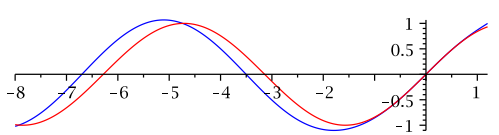

(a)

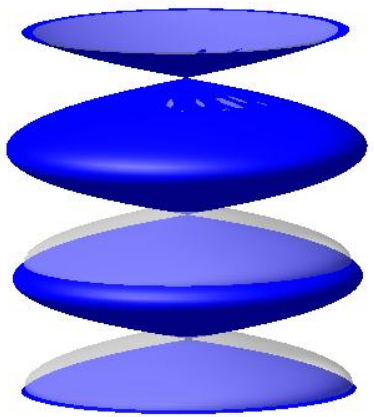

(b)

Figure 4. CMC $H=\frac{1}{4}$ : (a) Profile Curve $h(u),-8 \leq u \leq 1.2$, (b) Lorentz Surface of Revolution in $\mathbb{S}_{1}^{3}\left(\frac{1}{16}\right)$

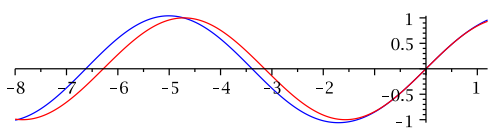

(a)

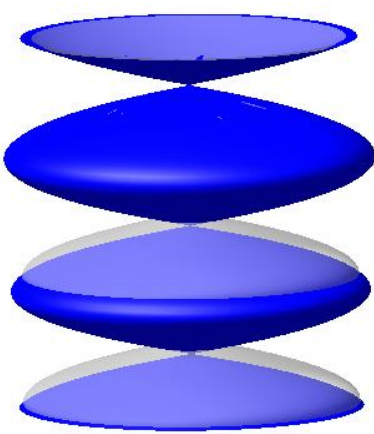

(b)

Figure 5. CMC $H=\frac{1}{8}$ : (a) Profile Curve $h(u),-8 \leq u \leq 1.2$, (b) Lorentz Surface of Revolution in $\mathbb{S}_{1}^{3}\left(\frac{1}{64}\right)$

\section{Minimal Lorentz Surface of Revolution in $\mathbb{S}^{3}\left(c^{2}\right)$}

In this section, we construct a minimal Lorentz surface of revolution in $\mathbb{S}_{1}^{3}\left(c^{2}\right)$ using the calculus of variation. Let us consider a surface of revolution which is obtained by rotating a timelike curve $x(t)$ in the $t x$-plane about the $t$-axis. The curve is required to pass through the points $\left(t_{1}, x_{1}\right)$ and $\left(t_{2}, x_{2}\right)$ as shown in Figure 8. Since $x(t)$ is a timelike curve, the squared infinitesimal arc-length shown in Figure 8

$$
d s^{2}=-(d t)^{2}+e^{2 c t}(d x)^{2}
$$

is negative. So, the infinitesimal arc-length $d s$ should be the proper time $\sqrt{-d s^{2}}$ which we will simply denote by $d s$. Thus, the area element $d A$ for a Lorentz surface 


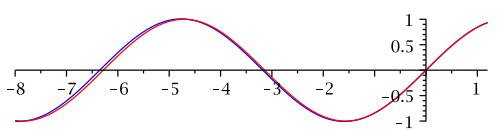

(a)

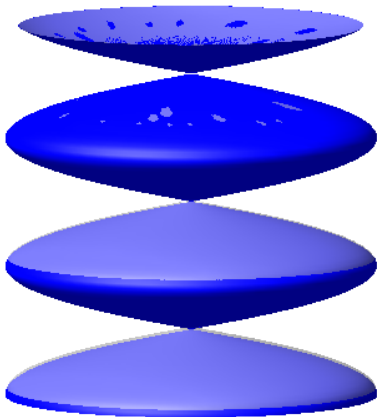

(b)

Figure 6. CMC $H=\frac{1}{64}$ : (a) Profile Curve $h(u),-8 \leq u \leq 1.2$, (b) Lorentz Surface of Revolution in $\mathbb{S}_{1}^{3}\left(\frac{1}{4096}\right)$

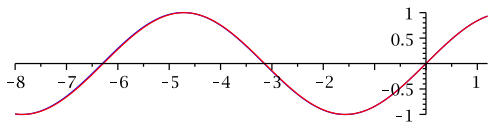

(a)

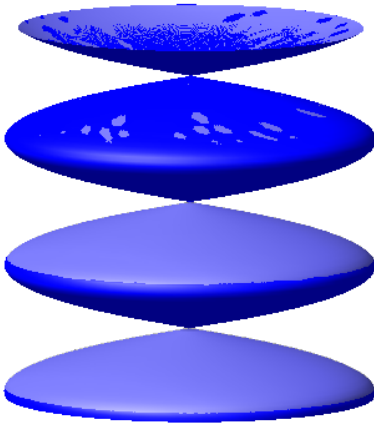

(b)

Figure 7. CMC $H=\frac{1}{256}$ : (a) Profile Curve $h(u),-8 \leq u \leq 1.2$, (b) Lorentz Surface of Revolution in $\mathbb{S}_{1}^{3}\left(\frac{1}{65535}\right)$

of revolution is given by

$$
\begin{aligned}
d A & =2 \pi x(t) d s \\
& =2 \pi x(t) \sqrt{(d t)^{2}-e^{2 c t}(d x)^{2}} \\
& =2 \pi x(t) \sqrt{1-e^{2 c t} \dot{x}(t)^{2}} d t,
\end{aligned}
$$

where $\dot{x}(t)=\frac{d x}{d t}$. The area functional is then

$$
J=\int_{t_{1}}^{t_{2}} 2 \pi x(t) \sqrt{1-e^{2 c t} \dot{x}(t)^{2}} d t .
$$




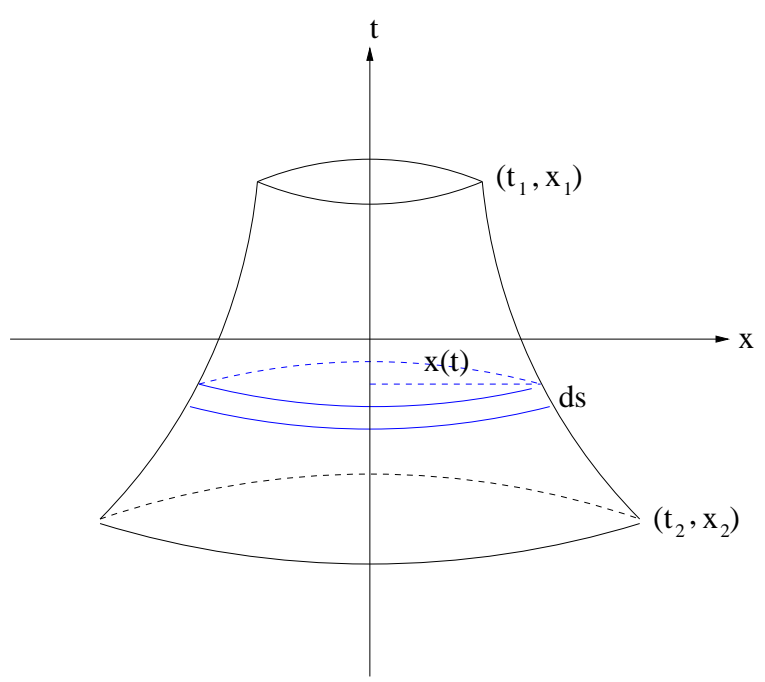

Figure 8. Surface of Revolution in $\mathbb{S}_{1}^{3}\left(c^{2}\right)$

Let ${ }^{5} f(x, \dot{x}, t)=x \sqrt{1-e^{2 c t} \dot{x}^{2}}$. Finding a critical point of the area functional is equivalent to solving the Euler-Lagrange equation (see [1] for example)

$$
\frac{\partial f}{\partial x}-\frac{d}{d t} \frac{\partial f}{\partial \dot{x}}=0 .
$$

The Euler-Lagrange equation (6.3) is equivalent to the second order nonlinear differential equation

$$
-1+e^{2 c t} \dot{x}^{2}+c x e^{4 c t} \dot{x}^{3}-2 x c e^{2 c t} \dot{x}-x e^{2 c t} \ddot{x}=0 .
$$

We require the timelike surface of revolution to be conformal. Applying the conformality condition (4.3) in terms of $x(t)$, the equation (6.4) simplifies to

$$
\ddot{x}+c\left(1+e^{2 c t} x^{2}\right) \dot{x}+x=0 .
$$

We solve this nonlinear differential equation numerically as it cannot be solved analytically. Figure 9 shows the profile curve $x(t)$ and the minimal Lorentz surface of revolution in $\mathbb{S}_{1}^{3}(1)$. For the numerical solution, we used the same initial conditions $x(0)=0$ and $\dot{x}(0)=1$ as before.

If $c \rightarrow 0$, then (6.4) becomes the equation of underdamped simple harmonic oscillator (4.7). Thus, as $c \rightarrow 0$ minimal Lorentz surfaces of revolution in $\mathbb{S}_{1}^{3}\left(c^{2}\right)$ also tend toward the timelike catenoid, the minimal timelike surface of revolution in $\mathbb{R}^{2+1}$. An animation of this limiting behavior of minimal Lorentz surfaces of revolution in $\mathbb{S}_{1}^{3}\left(c^{2}\right)$ is available at http://www.math.usm.edu/lee/tldsminimalanim.gif.

\footnotetext{
${ }^{5}$ The constant $2 \pi$ can be ignored since it makes no contribution to the solution of our variational problem.
} 


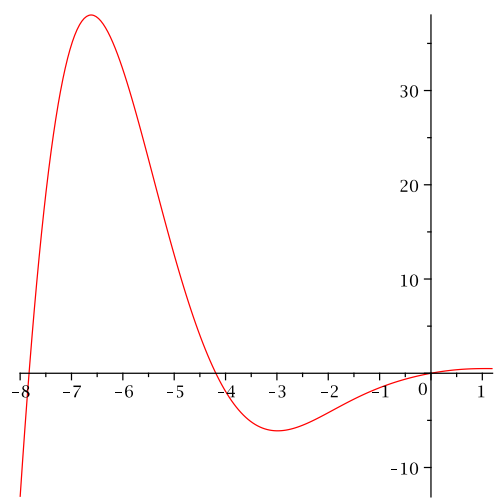

(a)

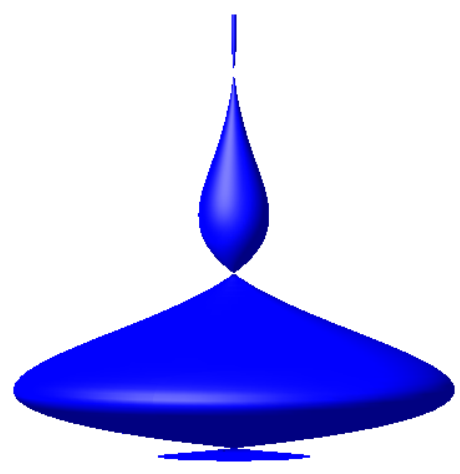

(b)

Figure 9. (a) Profile Curve $x(t),-8 \leq u \leq 1.2$, (b) Minimal Lorentz Surface of Revolution in $\mathbb{S}_{1}^{3}(1)$

\section{REFERENCES}

[1] Arfken, G., Mathematical Methods for Physicists, 3rd Edition, Academic Press, 1985.

[2] Hawking, S. W. and Ellis, G. F. R., The Large Scale Structure of Space-Time, Cambridge University Press, 1973.

[3] Blaine Lawson, H. Jr., Complete minimal surfaces in $S^{3}$, Ann. of Math. 92 (1970), 335-374.

[4] Lee, S., Timelike surfaces of constant mean curvature \pm 1 in anti-de Sitter 3 -space $\mathbb{H}_{1}^{3}(-1)$, Ann. Global Anal. Geom. 29 (2006), no. 4, 361-407.

[5] Lee, S. and Varnado, J. H., Timelike surfaces of revolution with constant mean curvature in Minkowski 3-space, Differential Geometry and Dynamical Systems 9 (2007), No. 1, 82-102.

[6] Lee, S. and Zarske, K., Surfaces of Revolution with Constant Mean Curvature in Hyperbolic 3-Space, Differential Geometry - Dynamical Systems 16 (2014), 203-218.

[7] O’Neill, B., Elementary Differential Geometry, Academic Press, 1967.

Department of Mathematics, University of Southern Mississippi,, Hattiesburg, MS 39401, USA

E-mail address: sunglee@usm.edu

Massachusetts Institute of Technology, Cambridge, MA 02139, USA

E-mail address: jacobm@mit.edu 